\title{
Spreading and Power Allocation for Multiple Antenna Transmission using Decorrelating Receivers
}

\author{
Dinesh Rajan, Elza Erkip and Behnaam Aazhang
}

\begin{abstract}
We propose a new scheme for multiple antenna transmission in the context of spread spectrum signaling. The new scheme consists of using shifted Gold sequences to modulate independent information on the multiple antennas. We show that this strategy of using Multiphase Spreading (MPS) on different antennas greatly improves the throughput over currently known spread-spectrum multipleantenna methods. We also find the optimal power allocation strategy among multiple transmit antennas for a fixed rate of channel state information, which might be provided via a feedback link, at the transmitter. We demonstrate the differences in optimal power distribution for maximizing capacity and minimizing probability of outage. When the transmission from the two antennas uses orthogonal spreading, we find that optimizing the power does not give much gain over the equal power transmission. However, when the transmissions are not orthogonal as in the case of MPS, then allocating power to maximize throughput gives considerable gain over equal power transmission. We also consider the effect of imperfections in the feedback channel on the optimal power allocation and show that our power allocation scheme is robust to feedback errors.
\end{abstract}

Keywords- Multiple antennas, CDMA, Power control, multiuser detection, Gold sequences, outage probability.

\section{INTRODUCTION}

Multiple antennas are vital in downlink communications of future cellular systems to achieve higher bit rates and better quality of services. Wideband code division multiple access (W-CDMA) has been universally accepted as the preferred multiple accessing scheme for the next generation cellular systems.

In this paper, we propose a new spreading method for multiple antenna transmission. We show that using different phases of the same spreading code on different antennas and modulating independent information results in higher throughput. In the future we refer to our proposed method as Multiphase Spreading (MPS). The main reason for using different phases of the same code is to maintain the total number of codes available per user to be constant for a fixed transmission bandwidth. Although MPS introduces additional multiuser interference, we show that it achieves satisfactory performance using simple multiuser detectors [1]. In this paper we focus our attention on two phase MPS for two transmit antennas. However, MPS can be extended

Dinesh Rajan is with the Department of Electrical Engineering, Southern Methodist University, Dallas, TX. Elza Erkip is with the Department of Electrical and Computer Engineering, Polytechnic University, Brooklyn, NY. Behnaam Aazhang is with the Department of Electrical and Computer Engineering, Rice University, Houston, TX. This work was supported in part by Nokia Corporation, Texas Advanced Technology Program under grants 1999-003604-0143 and 1999-003604-0075, and by NSF under grant ANI-9979465. to higher phases if there are more transmit antennas and the number of chips in the spreading code is substantially larger than the multipath spread of the channel.

It is well known that Gold codes have good crosscorrelation properties [2]. They are designed so that circularly shifted codes have low cross-correlation with the unshifted ones. We use this property to increase the throughput of the system without decreasing the number of users the system can support. We show that the increased throughput can alternatively be traded to get diversity gain.

Using different shifts of the same code is analogous to introducing multiple paths to the channel which can be resolved unambiguously at the receiver to get processing gains. If the same information is modulated on the two antennas using different shifts of the same code, the received signal is equivalent to a signal transmitted from one antenna and passed through a channel with multipath profile corresponding to the shifted sequence. However in MPS, we propose to modulate independent information over shifted spreading sequences. Of course, care should be taken to make sure the artificial multipaths introduced are not too close to one another or to the multipaths introduced by the channel. The difference between MPS and delay diversity is that the information modulated on the artificial multipaths introduced is the same in delay diversity while it is different in MPS.

Several ideas for exploiting the spatial dimension have been proposed in $[3,4]$ using special multi-element array (MEA) technology. Foschini et al. [3] focus their attention on 'quasi-static' channels which typically occur in wireless LAN environments. Their scheme utilizes a large number of transmit and receive antenna elements while our work relates to a downlink cellular system where the mobile has a single antenna and the base station has a small number of antennas.

If the total transmission power is constrained then the division of power between the different transmit antennas affects the achievable performance. We assume that the current channel state information (CSI) is known perfectly at the receiver. In the absence of any CSI at the transmitter, the optimum power distribution is uniform among the antennas for identical fading statistics. When perfect CSI is available at both the transmitter and receiver, optimum power allocation across antennas and time to maximize capacity [5] or to minimize probability of outage [6] has been investigated. We focus on BPSK modulation and find the 
optimal power allocation for MPS transmission, to maximize capacity and minimize probability of outage when partial CSI is available at the transmitter. This partial knowledge is gathered from constant rate feedback from the receiver to the transmitter. Our optimal power allocation strategy is in agreement with [7] where the problem of optimizing the power to maximize the mutual information between the input and output is investigated. We also consider the effect of feedback errors on the proposed power allocation methods.

The diversity gain achieved by a system is related to the exponent of SNR in the probability of error expression [8]. A system having infinite diversity converts a Rayleigh fading channel to an additive Gaussian channel. For a system with $m$ transmit and $n$ receive antennas the maximum achievable diversity is $m n$. Space-time codes [8] attempt to achieve full diversity gain for multiple transmit and receive antennas by joint coding over the transmit antennas. However, as we will illustrate in Section IV the performance of Alamouti space time codes [9] in a slowly fading channel is inferior to MPS for low SNR values. Also, when used for multiple users space time codes require an explicit multiuser modulation scheme whereas MPS inherently has multiuser capability. MPS makes only minor changes to the modulation layer and can be easily implemented in present and future cellular systems; MPS is essentially a space-time modulation method for CDMA systems.

The remainder of the paper is organized as follows. In Section II we introduce the concept of MPS and explain it in detail, followed by its performance analysis in Section III. The performance of MPS in the presence of convolutional codes and its comparison with space-time codes is given in Section IV. Section V explores optimum power allocation strategies with partial CSI at the transmitter. Finally, we present our conclusions in Section VI.

\section{Multiphase Spreading}

In this section we introduce the concept of MPS while confining ourselves to a specific modulation scheme - binary phase shift keying (BPSK). The analysis can be extended to higher level modulation schemes in a straightforward manner. We consider the downlink case where the base station sends independent information to different mobiles. We focus our attention on one user for our analysis although the model incorporates the interference generated by the other users within a given cell. The interference from other cell users is ignored. We assume the mobile receivers can track the fading coefficients corresponding to signals coming from different antennas. This is a reasonable assumption for channels with low Doppler shifts.

We confine ourselves to direct sequence CDMA and use Gold codes for spreading purposes. In our scheme, the base station transmits two independent bit streams for each user through the two antennas, using different phases, or shifted versions, of the same spreading code. Hence we maintain the total number of spreading codes available for different users the same as in the single antenna case. Also as the spreading length is kept unchanged, we maintain the same bandwidth as in a single transmit antenna. The MPS modulation scheme is illustrated in Figure 1. The transmitted signal on antenna $j$ at time $i$ is given by

$$
X_{j}[i]=\sum_{k=1}^{K} P_{k j} s_{k j} b_{k j}[i],
$$

where $P_{k j}, s_{k j}, b_{k j}[i]$ denote respectively the power, the spreading code and the bit belonging to the $k^{\text {th }}$ user modulated on the $j^{t h}$ antenna at time $i$ and $K$ denotes the total number of users. Note that MPS with, say, two antennas will have $s_{k 1}$ as a Gold code, $s_{k 2}$ as the same Gold code shifted by a predetermined amount. The amount of shift is chosen to be larger than the multipath spread of the channel: This condition is needed in order to be able to distinguish between multipaths from one transmit antenna and the information from the other antenna. We assume that the amount of shift in the spreading code is the same for all users.

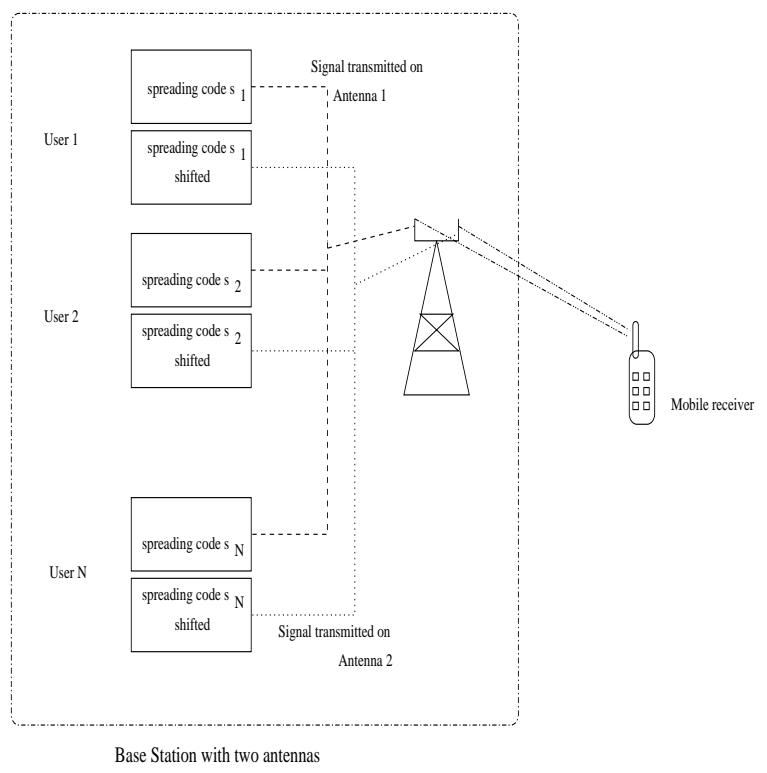

Fig. 1. Multiphase Spreading

We assume the total transmit power allocated to a particular mobile is $P$. When no CSI is available at the transmitter, since fading distributions are identically distributed, the bits on both the antennas receive half the power. Thus, $P_{k 1}=P_{k 2}=P / 2$. When finite rate CSI about antenna fading coefficients is available at the transmitter, the power allocation between antennas can be optimized. Such optimization is carried out in Section V for two different optimization criterion namely, maximizing capacity and minimizing probability of outage.

For two antennas at the base station and a flat fading channel, the received signal at a particular mobile (assuming that the mobile has only one receive antenna) is given by

$$
Y[i]=\sum_{j=1}^{2} X_{j}[i] A_{j}[i]+z[i]
$$


where $A_{j}[i]$ is the identically distributed fading amplitude at time $i$ between antenna $j$ and the mobile, $z[i]$ is the additive white Gaussian noise with variance $\sigma^{2}$.

At the mobile receiver, the signal first passes through a bank of chip matched filters. Although different users are chip synchronous with one another, since their spreading codes are not orthogonal we have multiple access interference (MAI). This MAI is mitigated by a multiuser detector at the receiver. In this paper, we analyze the system using a simple one shot decorrelator detector [1]. However we expect that the performance of MPS will be similar with other detectors such as MMSE. In fact since the MMSE receiver optimally reduces the effect of noise and multiple access interference, the performance of MPS will be better than what is achieved by a one shot decorrelator. In implementing the decorrelating receiver, the mobiles need to have information regarding the code correlation matrix which could be passed to the receivers from the base station when a new connection is established or hand-off from a neighboring cell takes place. ${ }^{1}$

\section{Performance Analysis of MPS}

This section contains performance analysis of MPS and comparison with other multiple antenna methods. The performance metrics we consider are capacity and probability of outage [6]. We assume a BPSK modulation, enabling us to convert the underlying additive Gaussian noise channel to a binary symmetric channel (BSC) whose crossover probability is given by the bit error rate of the particular multiple antenna strategy under question. The capacity, or throughput, is better suited for fast fading scenario whereas probability of outage captures achievable rates and frame error rates in a slow fading environment. We first start with a brief discussion of some transmit antenna methods proposed for the next generation of cellular systems.

\section{A. Comparison of various transmit antenna diversity schemes}

In this section, we present an information theoretic analysis with no constraint on modulation of three different transmit diversity schemes that have been proposed for the next generation cellular systems. These schemes are orthogonal transmit diversity (OTD) [11], time switched transmit diversity (TSTD) [12] and selection transmit diversity (STD) [13]. A block diagram representation is given in Figure 2.

Without loss of generality, we consider two transmit antennas and one receive antenna for the analysis. In OTD, each user uses two different orthogonal spreading codes for

\footnotetext{
${ }^{1}$ Although all users need to have information about the new code correlation matrix, it suffices for the base station to only broadcast the spreading code (or just the new row of the code correlation matrix) of the new user. Using the other spreading codes which are already known, each mobile can compute the new code correlation matrix. One of the major concerns in using multiuser detectors is the computational complexity. However, we feel that with improved VLSI architectures simple decorrelating detectors can be easily implemented in the mobile handset. Tradeoffs between complexity and performance of various single user and multiuser detector architectures in the mobile handset are investigated in [10].
}

transmission on the two antennas. To maintain the number of spreading codes available for each user the same as in single antenna case, the length of the spreading code is doubled, that is the number of chips is doubled. Also, to maintain the transmission bandwidth the same as in single antenna case, the bit period needs to be doubled. However, since two antennas are used for transmission simultaneously, we are able to support the same information rate for a fixed channel coding scheme. In TSTD, the same spreading code is used on both the antennas but at each time instant only one antenna is used. The transmitter switches between the antennas in a preassigned manner. In STD (also called maximal selection) the transmitter uses feedback information from the mobile. This feedback consists of one bit of information and indicates which of the two antennas has lower attenuation at every symbol instant. The transmitter then chooses the antenna with lowest attenuation to transmit the information. Note that the antenna with lowest attenuation between the base station and the mobile could be different for different mobiles. In practice the fading coefficient of the channel would change much faster than the feedback rate and hence the performance of any practical feedback scheme would be much worse than the idealized one that we present here. However we show that the MPS strategy with no feedback has better performance than even this hypothetical ideal feedback situation for a wide range of SNR values.

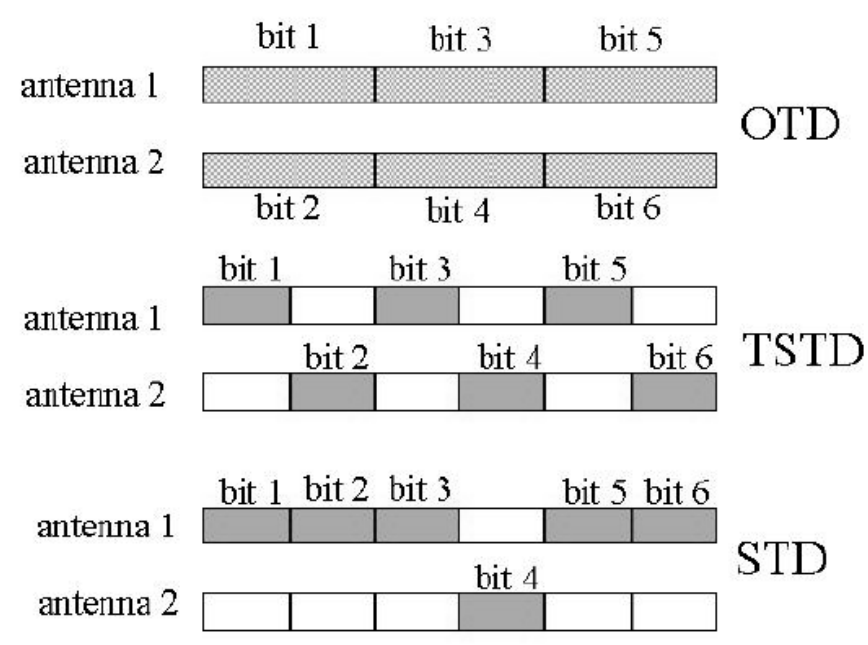

Fig. 2. Structure of data transmission for different orthogonal transmission schemes

For the analysis below, we focus on a single transmitterreceiver pair and consider the downlink transmission in a system that has two transmit and one receive antennas. The received signal $Y[i]$ at time $i$ is given by (2). The signals $X_{1}[i]$ and $X_{2}[i]$ depend on the transmit diversity structure used.

We can easily obtain the unconstrained modulation capacity of OTD and TSTD to be the same as that of the single antenna which is given by

$$
C_{\text {single }}=\frac{1}{2} E\left[\log \left(1+\left|A_{1}\right|^{2} S N R\right)\right] .
$$


The capacity of STD is higher as expected and is given by

$$
C_{S T D}=\frac{1}{2} E\left[\log \left(1+\max \left(\left|A_{1}\right|^{2},\left|A_{2}\right|^{2}\right) S N R\right)\right],
$$

where the expectation is over the fading states $A_{1}$ and $A_{2}$. A more detailed information theoretic analysis of multiple antenna schemes can be found in [14].

The capacity analysis of multiple antenna Gaussian channels [5] reveals that with no CSI at the transmitter, it is optimum to send independent information on the different antennas. One way to do this through CDMA without increasing the bit period is to have orthogonal spreading codes on the different antennas. However this reduces the number of degrees of freedom available for other users. Another strategy is to use the same spreading code on both antennas which necessitates a complex joint decoder structure. The MPS scheme keeps the bandwidth and number of codes per user constant, and uses the shifts of the same spreading code to send independent information on different antennas. We essentially make use of a "multipath" type of diversity where we control the delay of the multipath components and the information modulated on them. Although this introduces extra interference, we will observe that it can be mitigated by a simple linear multiuser detector.

\section{B. Throughput}

The study in Section III-A showed that OTD and TSTD have the same capacity as a single transmit antenna. Although this result was arrived at using the assumption of a Gaussian channel with no specific modulation, it is valid whenever modulation schemes used on the antennas are the same and the fading process over the two antennas have identical distributions. Note that this result does not depend on the correlation between the fading distributions of the antennas. Also, next generation cellular systems are likely to have some variation of STD as one of the advanced modes of operation. Hence for the remainder of the paper, we only compare MPS with single antenna and STD.

When multiple users are present as in (1) and a decorrelating detector is used at the receiver, the probability of bit error in the single antenna [1] and STD cases are given, respectively, by

$$
\begin{aligned}
P_{e-\text { single }} & =Q\left(\frac{\left|A_{1}\right| \sqrt{P}}{\sigma \sqrt{\left(R^{-1}\right)_{11}}}\right) \\
P_{e-s t d} & =Q\left(\frac{\max \left(\left|A_{1}\right|,\left|A_{2}\right|\right) \sqrt{P}}{\sigma \sqrt{\left(R^{-1}\right)_{11}}}\right),
\end{aligned}
$$

where $s_{k}$ is the spreading code of the $k^{t h}$ user, $R_{k l}=s_{k}^{t} s_{l}$, $k, l=1,2, \ldots, K$ is the $k l^{t h}$ entry of the code correlation matrix $R$ and $P$ is the power allocated to the particular mobile. Note that $\mathrm{R}$ is a $K \times K$ matrix where $\mathrm{K}$ is the number of users. For MPS with one shot decorrelator, the bit error probability for the two independent bit streams transmitted on the two antennas are given by

$$
\begin{aligned}
& P_{e 1}=Q\left(\frac{\left|A_{1}\right| \sqrt{P / 2}}{\sigma \sqrt{\left(\tilde{R}^{-1}\right)_{11}}}\right) \\
& P_{e 2}=Q\left(\frac{\left|A_{2}\right| \sqrt{P / 2}}{\sigma \sqrt{\left(\tilde{R}^{-1}\right)_{22}}}\right) .
\end{aligned}
$$

Note that in the two phase MPS scheme $\tilde{R}$ [1] incorporates the shifted as well as the unshifted spreading codes. If the receiver is synchronized to the primary stream of user $k$, then the signals to be considered in computing $\tilde{R}$ are: (a) One desired primary stream of user $k$. (b) Two asynchronous ${ }^{2}$ signals from the secondary stream of user $k$. (c) $K-1$ synchronous signals from the primary streams of the other $K-1$ users and (d) 2(K-1) signals from the asynchronous secondary streams of the $K-1$ other users. Hence, the size of $\tilde{R}$ is $3 K \times 3 K{ }^{3}$

We compute the throughput of each system by modeling the channel between each antenna and the mobile as a BSC with crossover probabilities given by the appropriate probabilities of error. The throughput in the single antenna and STD cases are given by

$$
\begin{aligned}
C_{\text {single }} & =E\left[1-H\left(P_{e-s i n g l e}\right)\right] \\
C_{\text {std }} & =E\left[1-H\left(P_{e-s t d}\right)\right]
\end{aligned}
$$

where $H(p)$ is the binary entropy function [15] and the expectation is over the fading process. In MPS, the noise processes in the two BSC's are derived from the same Gaussian noise process of the channel and hence are correlated. However, for the typical range of correlations associated with MPS, the throughput of the system is almost equal to the throughput assuming the BSC noises are independent (see Appendix A). For analytical exposition, we use the throughput expression assuming the noises are independent. Since independent bits are transmitted through the two antennas, the throughput of the system is given by the sum of two respective BSC throughputs with crossover probabilities of $P_{e 1}$ and $P_{e 2}$ and can be expressed as

$$
C_{m p s}=E\left[2-H\left(P_{e 1}\right)-H\left(P_{e 2}\right)\right] .
$$

If the fading processes are identically distributed, the throughput can be simplified as

$$
C_{m p s, \text { identical fading }}=2 E\left[1-H\left(P_{e 1}\right)\right] .
$$

We plot the throughput for the single antenna, STD and MPS in Figure 3a for 5 users using Gold codes of length 31. In our numerical evaluations, we model the fading processes $A_{1}$ and $A_{2}$ as independent and identical Rayleigh

\footnotetext{
${ }^{2} \mathrm{We}$ are using asynchronous here to refer to the signal on shifted spreading sequences.

${ }^{3}$ The size of $\tilde{R}$ will be $3 K \times 3 K$ even if the different users use different shifts of their spreading code on their different antennas. However, we assume that all users shift their respective spreading codes in an identical manner.
} 
distributions. Figure 3a shows that MPS performs very well at high SNR. Although the performance of MPS deteriorates for low SNR's we feel that in the range of SNR's required for normal operating bit error rates, the performance is satisfactory. We also show the performance of MPS, STD and single antenna transmission for different number of users in Figure $3 \mathrm{~b}$. It is clear from Figure $3 \mathrm{~b}$ that the performance of MPS does not reduce much with increasing number of users; improved MPS performance is possible with increasing number of users if a 'better' multiuser detector (like MMSE) is used [1].

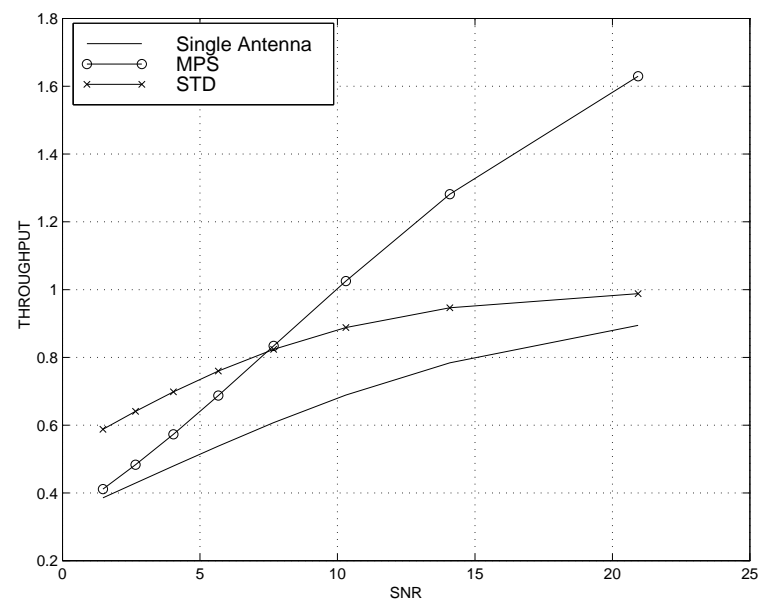

a

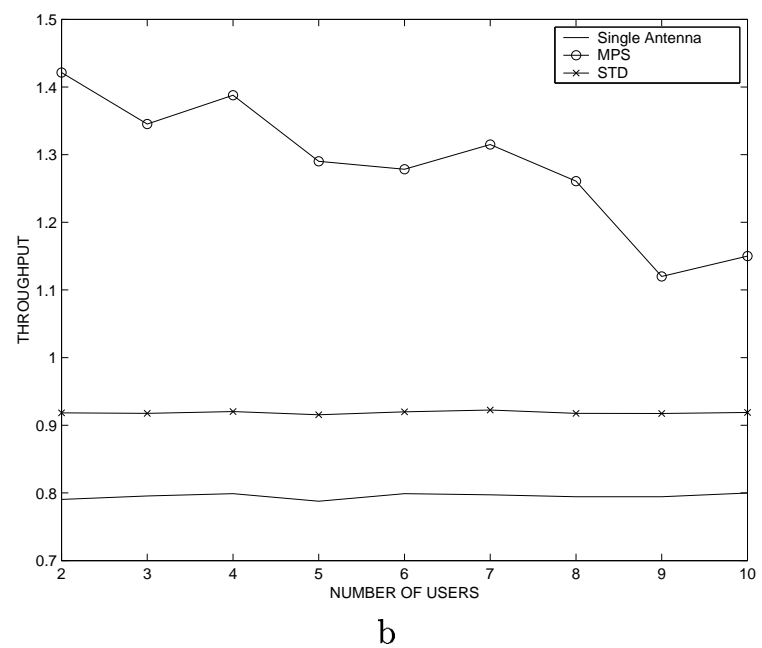

Fig. 3. Throughput (in bits/channel usage) of MPS, STD and single antenna a) versus SNR for 5 users b) versus number of users $K$ for SNR $=11 \mathrm{~dB}$. Gold code length 31, Rayleigh fading channel

The fact that MPS is better than even STD could be surprising at first. However this is in line with the arguments provided in [16] where it is proved that higher capacity is possible by having $N$ users at spatially different locations than having one user of $N$ times the power. Although the analysis in [16] was for the uplink scenario, we could consider the two antennas at the base station to represent different users with powers $P / 2$. This would be better than having one user at power $P$ on one of the antennas alone. Also using BPSK modulation scheme limits STD from achieving rates greater than $1 \mathrm{bit} /$ transmission, whereas MPS can transmit up to 2 bits/transmission. Although one could transmit 2 bits/transmission using STD and then compare the performance with MPS, this involves increasing the modulation from BPSK to QPSK in STD. Correspondingly, we could also increase the modulation of MPS to QPSK and transmit 4 bits/transmission. We maintain the same modulation for all schemes employed in order to have a fair comparison. Analyzing the performance of STD and MPS for different modulation schemes (like $M$ PSK and QAM) is beyond the scope of this paper and should be investigated in future research.

\section{Probability of outage}

The probability of outage [6], is given by the probability that the instantaneous rate falls below some specified rate $r$ and hence the probability that data transmission at rate $r$ is not possible. The outage probability can also be thought of as the frame error rate for transmission at rate $r$ when a strong channel code is employed. The probability of outage for MPS can be expressed as

$$
P_{m p s, o u t}=\operatorname{Pr}\left(\left[2-H\left(P_{e 1}\right)-H\left(P_{e 2}\right)\right]<r\right) .
$$

We compare (11) to the probability of outage for the single antenna and STD under independent Rayleigh fading on the two antennas. Note that for $r \geq 1$, single antenna and STD have $P_{\text {out }}=1$, since the maximum throughput of single antenna and STD are 1. However, MPS can still support rates higher than 1 bit/transmission which is clearly evident from Figure 3. For $r<1$, STD has the advantage of knowing the best antenna and thus being less susceptible to variations. However, this comes at the expense of 1 bit of feedback about the channel state from the mobile receiver.

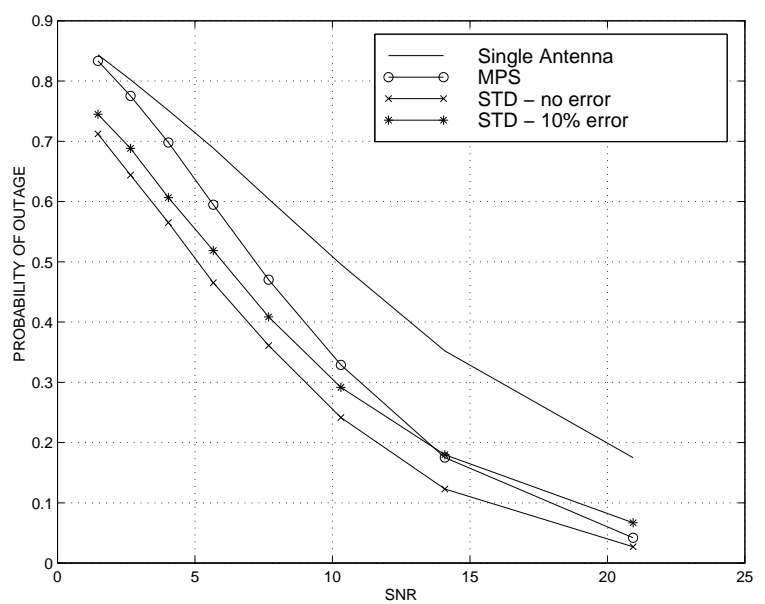

Fig. 4. Probability of outage for rate $=0.9$, Rayleigh fading, Gold code of length 31

In order to provide a comparison for probability of outage and study the sensitivity of STD to errors in the feedback, in Figure 4, we plot $P_{\text {out }}$ vs SNR for $r=0.9$ for single antenna, STD, STD with $10 \%$ error (feedback channel is assumed to be a BSC with crossover probability 0.1 ) and MPS. We observe that STD is quite susceptible to feedback 
errors and for high SNR, MPS has better performance than STD with feedback errors.

\section{CODED MPS}

\section{A. MPS with convolutional coding}

In the previous section we showed that MPS is capable of supporting high rates of information transmission by modulating independent information over different antennas. Alternatively, we can keep the incoming data rate constant and use the different phases of the spreading code to improve the probability of bit error and provide diversity. We consider a simple 'outer' coding scheme in which the coded data that was transmitted in the single antenna case is passed through a rate $1 / 2$ outer convolutional encoder. In general one could combine the outer code with the inner one and replace the channel code in single antenna of rate $k / n$ with a combined code of rate $k / 2 n$ which is illustrated in Figure 5. The resulting coded bit stream is multiplexed and sent over the two different antennas using MPS.

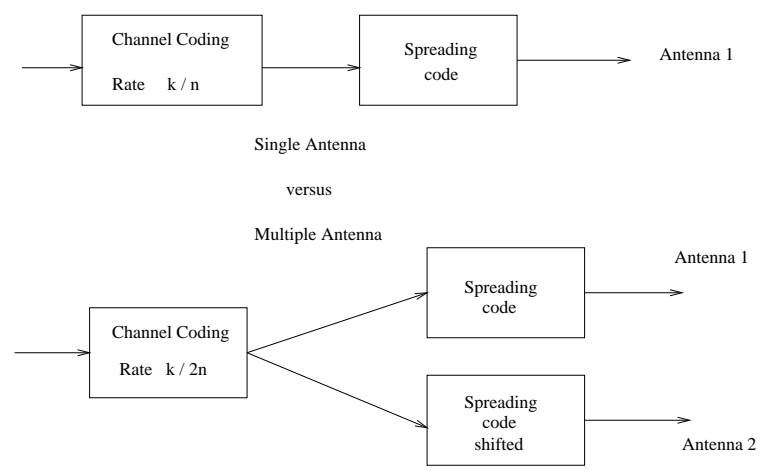

Fig. 5. Channel Coding with MPS

We have simulated the system using a rate $1 / 2$ convolutional encoder with generator matrix [23 35] for both the inner and outer coding [2]. The single antenna and STD simply use the inner convolutional code. The plot of bit error rates for coded MPS along with single antenna and STD are given in Figure 6. The results indicate that MPS performance is superior to the single antenna case. This shows that the increased spatial dimensionality due to the presence of multiple antennas can also be used to provide stronger channel coding. Also, when compared with STD, MPS performs well for high SNR. When we introduce feedback errors to STD, the performance deteriorates and bit error rate falls below MPS even for lower SNR's. Thus, similar to Section III-C, we conclude that STD is quite susceptible to errors in the feedback link.

\section{B. Comparison with Alamouti's space-time codes}

In [9], Alamouti proposed a space time coding scheme that achieves full diversity when transmitting 2 bits in two uses of the channel using two transmit antennas. In Alamouti's [9] scheme, the transmitted signal on the two anten-

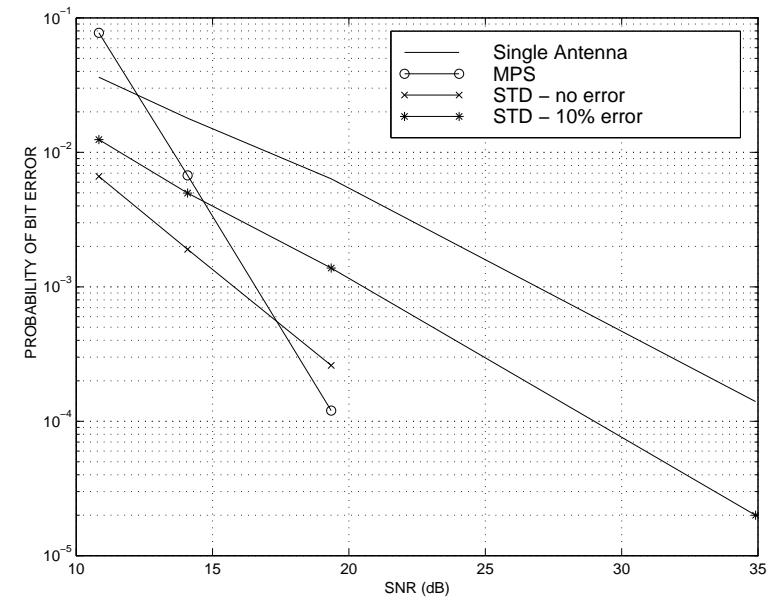

Fig. 6. Bit Error Rate versus SNR for rate $1 / 2$ outer coding in MPS. Rayleigh fading, Gold code length 31, 5 users.

nas at two consecutive instants of time are given by ${ }^{4}$

$$
\begin{aligned}
& X_{1}[i]=b_{i} \quad ; X_{1}[i+1]=b_{i+1} \\
& X_{2}[i]=-b_{i+1} ; X_{2}[i+1]=b_{i}
\end{aligned}
$$

It is assumed that the channel between the antennas and the receiver is constant over two symbol periods. Hence each bit effectively passes through a channel with fading amplitude $\left|A_{1}\right|^{2}+\left|A_{2}\right|^{2}$. Assuming the two phases of the spreading code in MPS are almost orthogonal, if we had an 'outer' code as a rate $1 / 2$ repetition code, then each bit in coded MPS would experience a fading amplitude $\left|A_{1}\right|^{2}+\left|A_{2}\right|^{2}$. Hence, its performance should be similar to that of Alamouti's code. However, MPS with (nonrepetitive) convolutional coding will perform better than MPS with repetitive coding and Alamouti's scheme at low SNR because of the coding gain of the convolutional coding. At high SNR the diversity gain of Alamouti's space-time code becomes more significant and compensates for the low coding gain. Our intuition is confirmed by the simulations as shown in Figure 7, which contains a BER comparison of Alamouti space-time code and MPS with a outer channel code of rate $1 / 2$ for a slow fading channel using Jakes' channel model. A convolutional code of constraint length 3 (generator matrix [5 7]) was used [2] which is closer to the memory of 2 inherent in Alamouti scheme. Hence we conclude that coded MPS is more suitable for low rate applications.

\section{Optimal Power Allocation}

\section{A. Allocation for throughput maximization}

In this section we will observe how the one bit feedback of the STD scheme can be used in the context of MPS. Recall that for STD the power allocated to the antennas is either $[P, 0]$ or $[0, P]$ depending on the feedback, whereas for MPS it is always $[P / 2, P / 2]$. Assuming this one bit of feedback is also available to MPS, we can find the optimum

\footnotetext{
${ }^{4}$ Since we are assuming BPSK, the complex conjugation is omitted in $X_{2}$.
} 


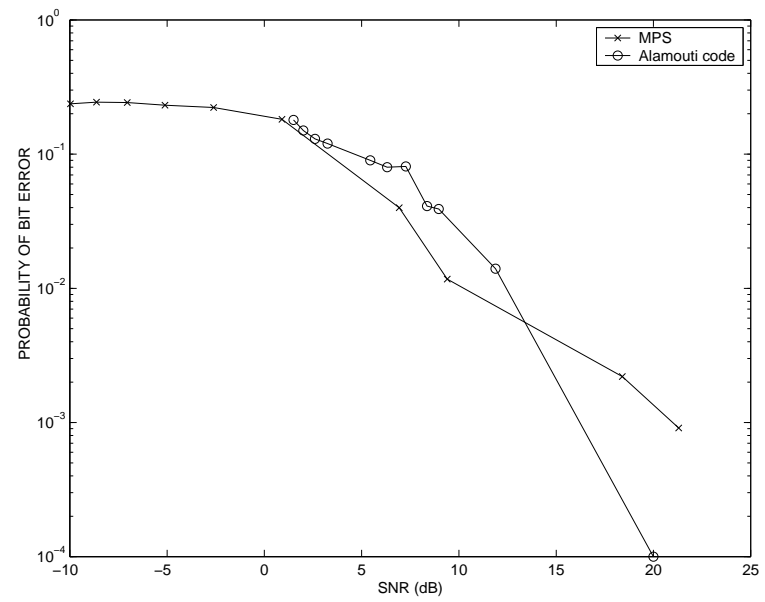

Fig. 7. Bit Error Rate of Alamouti space-time code and MPS, Jakes channel model $-10 \mathrm{~km} / \mathrm{hr}$, Gold code length $=31$.

power allocation $[\alpha P,(1-\alpha) P], 0 \leq \alpha \leq 1$. Note that this power allocation does not allow for waterfilling in time as in [17], it simply distributes the power among antennas. We consider the case when this one bit of feedback informs the transmitter about which antenna has lower attenuation, as in STD, although other forms of feedback are also possible [7]. Note that STD corresponds to $\alpha=1$ or 0 in the above formulation, whereas the no-feedback MPS has $\alpha=1 / 2$. For this optimization we assume the modulation is restricted to BPSK. It was shown in [7] that with no restriction on modulation, STD is optimal with the above feedback strategy. However, we will observe that fixing the modulation scheme results in power being allocated on both antennas.

For illustration purposes, we initially consider the case in which the spreading codes used on the two antennas as well as the spreading codes of different users are orthogonal to one another. This will form an upper bound on MPS system performance. Independent bits are modulated on different antennas. Assuming a synchronous system, other users do not contribute any MAI to the user of interest and the total throughput is given by

$$
\begin{aligned}
C_{p a, \text { orth }}= & E\left[2-H\left(Q\left(\left|A_{1}\right| \sqrt{\frac{\alpha P}{\sigma^{2}}}\right)\right)-\ldots\right. \\
& \left.H\left(Q\left(\left|A_{2}\right| \sqrt{\frac{(1-\alpha) P}{\sigma^{2}}}\right)\right)\right],
\end{aligned}
$$

where $\alpha$ is the power allocated to the 'good' antenna based on the one bit feedback. The optimum value of $\alpha$ depends on the operating SNR of the system and is shown in Figure $8 \mathrm{a}$ for independent fading between the two antennas. The numerical results are obtained with Rayleigh fading on the antennas. The throughput corresponding to optimal alpha is given in Figure 8b. We observe that for low SNR's the optimizing $\alpha$ is 1 , corresponding to STD. Optimal $\alpha$ is a decreasing function of SNR, converging to 0 as $S N R \rightarrow \infty$. This can be explained by the fact that the incremental gain in throughput for incremental power

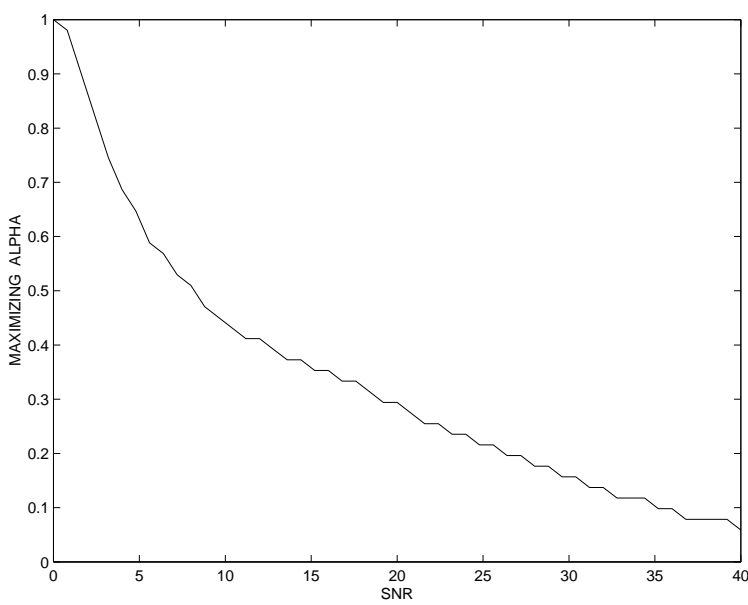

a

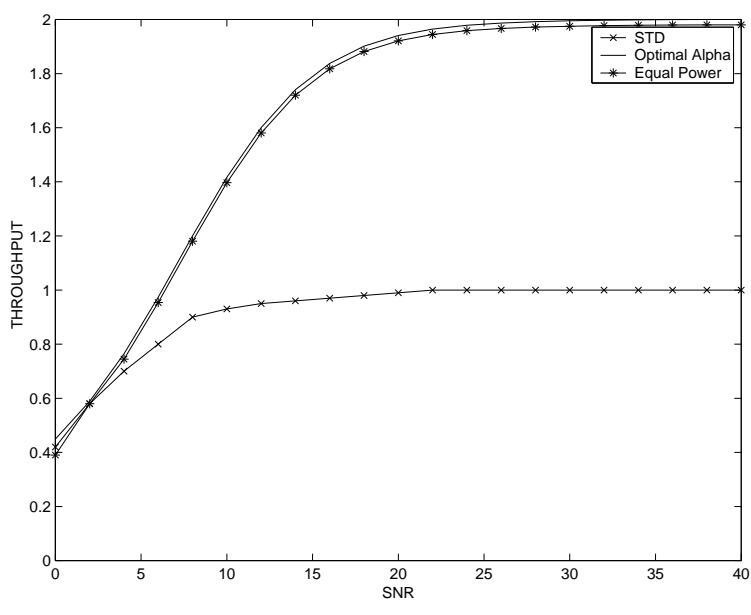

$\mathrm{b}$

Fig. 8. Orthogonal transmission on two antennas, single user system, BPSK modulation, 1 bit CSI at transmitter. a) Optimal power allocation between antennas b) Throughput under optimal power allocation, equal power allocation and STD.

on a particular antenna reduces as the total power on that antenna increases. Since the SNR versus throughput curve levels off for high SNR, once we put enough power on the 'better' antenna to get a throughput close to one, we will get more utility by allocating any extra power to the 'worse' antenna. We also observe that in general even though the maximizing $\alpha$ is different from $1 / 2$, the performance obtained by $\alpha=1 / 2$ (as suggested by MPS) is very close to optimal. Of course, choosing $\alpha=1 / 2$ requires no feedback, and hence is a lower complexity strategy.

The above analysis can be easily extended to incorporate MPS, which uses non-orthogonal spreading sequences across users. Assuming a decorrelating detector is used at the receiver to mitigate the MAI, the throughput is given by

$$
\begin{aligned}
C_{\text {pa,non-orth }=}= & E\left[2-H\left(Q\left(\left|A_{1}\right| \sqrt{\frac{\alpha S N R}{\left(\tilde{R}^{-1}\right)_{11}}}\right)\right)-\ldots\right. \\
& \left.H\left(Q\left(\left|A_{2}\right| \sqrt{\frac{(1-\alpha) S N R}{\left(\tilde{R}^{-1}\right)_{22}}}\right)\right)\right], \quad(15)
\end{aligned}
$$


where $\tilde{R}$ is the code correlation matrix of MPS as in (7).

Using the simulation parameters of Section III, (Figures 3 and 4 ) we plot the optimal $\alpha$ that maximizes $C_{p a, n o n-o r t h}$ with respect to SNR. The optimal $\alpha$ is illustrated in Figure 9a as the "no feedback error" curve. Note that similar to orthogonal transmission for Figure 8a, optimum $\alpha$ is a non-increasing function of SNR.

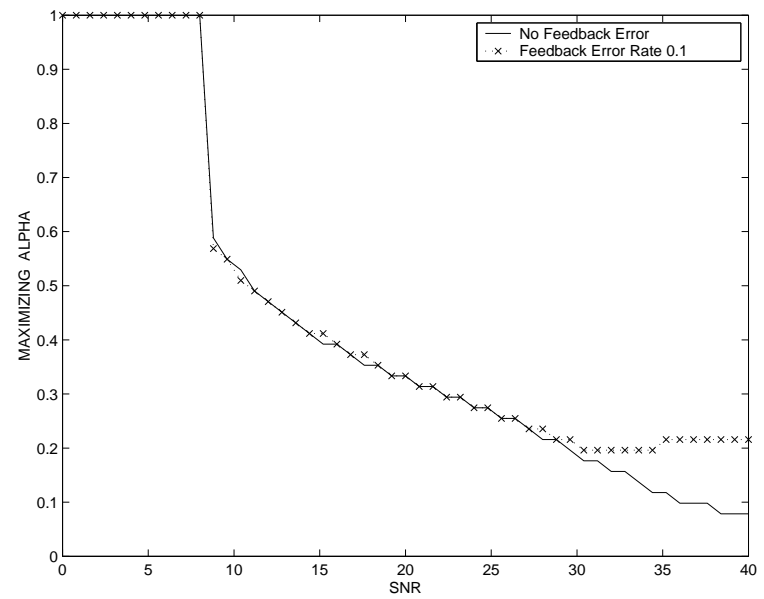

a

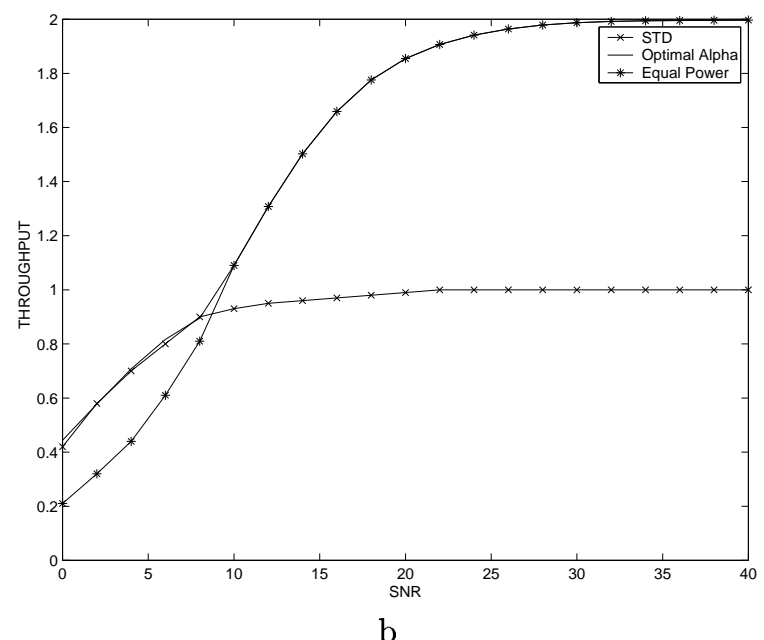

Fig. 9. Performance of MPS with 1 bit CSI at transmitter. a) Optimal power allocation for throughput maximization b) Throughput of MPS with optimal power allocation, MPS with equal power sharing and STD. Fast fading channel, BPSK modulation, Gold code length 31.

The optimal throughput as a function of SNR is shown in Figure 9b. The results are slightly different than in the orthogonal case: For low SNR values, the optimal power allocation is almost equal to STD and is superior to the equal power transmission of no feedback MPS. The small difference between STD and MPS with $\alpha \simeq 1$ occurs because of the way we have modeled the other users within the cell. All users are assumed to operate under the same scheme, whether it be STD or MPS, and the spreading codes used for the two antennas in STD and MPS result in different correlation matrices. ${ }^{5}$ For high SNR, the optimal

\footnotetext{
${ }^{5}$ If for example, $\alpha=0.99$, then the power allocated to the "good" antenna equals 0.99 which is almost the same as the power allocated
}

throughput is almost the same as the throughput assuming MPS with equal power allocation to the two antennas.

The main difference between power allocation in the case of 1 bit feedback for orthogonal and non-orthogonal systems is that for orthogonal systems using equal power allocation on the two antennas is near optimal for all SNR values. Hence, there is no need for feedback about the channel state. However, in non-orthogonal systems equal power allocation on the two antennas is near optimal only for high SNR values. For low SNR values, there is significant gain in optimizing the power allocation. For orthogonal systems, changing the transmit power on one antennas only affects the available power for the other antenna; however, for non-orthogonal systems, changing the power on one antenna not only affects the available power for the other antenna, but also the amount of interference between the two antennas. Hence, optimal power allocation gives more gain over equal power allocation in non-orthogonal case.

Since the CSI at the transmitter is received through a noisy feedback channel, we model the feedback channel to be a BSC. The optimum power allocation $\alpha$ versus SNR is shown in Figure 9a for no feedback errors and for $10 \%$ feedback error rate. Since the optimum $\alpha$ does not change considerably with increasing error rates in the feedback channel, the optimum MPS throughput is robust to reasonable feedback errors. ${ }^{6}$

\section{B. Allocation for probability of outage minimization}

In the above analysis, the power distribution was optimized to maximize the throughput. Alternatively, we can find optimal power distribution to obtain minimum probability of outage for any rate $r$. In other words the optimization problem now is

$$
\begin{aligned}
\min P r & \left(\left[2-H\left(Q\left(\left|A_{1}\right| \sqrt{\frac{\alpha S N R}{\left(\tilde{R}^{-1}\right)_{11}}}\right)\right)-\ldots\right.\right. \\
& \left.\left.H\left(Q\left(\left|A_{2}\right| \sqrt{\frac{(1-\alpha) S N R}{\left(\tilde{R}^{-1}\right)_{22}}}\right)\right)\right]<r\right)
\end{aligned}
$$

The optimum value of $\alpha$ in the above optimization depends on rate $r$ and is illustrated in Figure 10 for the simulation parameters of Section III. Figure 10 also contains the comparison of optimal power allocations for different values of $r$.

We observe that for $r$ lower than the maximum possible throughput of one antenna (in this case 1), it is better to allocate most of the power to the better antenna. Hence for $r<1$, STD is near optimal. For $r \geq 1$ we notice a

in STD. However, when all users are using STD, the code correlation matrix is of size $K \times K$ and if all users are using MPS, we have a different code correlation matrix (size $3 K \times 3 K$ ). Also when $\alpha=0.99$ in MPS system for a particular user, the other users might have lower values of $\alpha$ and hence their asynchronous streams contribute more interference than in STD case. Hence the performance of MPS with $\alpha \simeq 1$ is different from STD.

${ }^{6}$ Although there will be a drop in throughput with feedback errors compared to no feedback error, the optimal power allocation strategy in both cases will approximately be the same. 


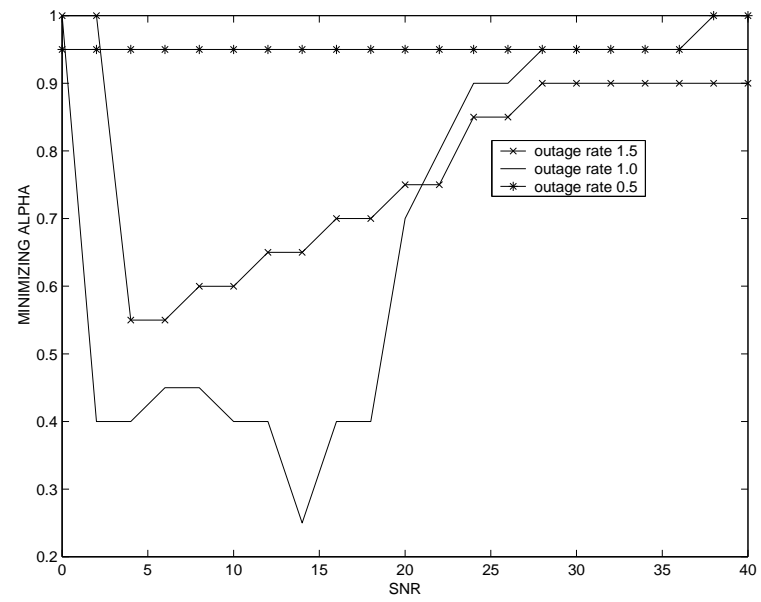

Fig. 10. Power allocation for outage minimization in MPS. Gold code length 31, Rayleigh fading, 1 bit CSI at transmitter.

non-monotonic dependence of $\alpha$ on SNR. For low SNR, the optimum $\alpha$ is 1 . As SNR increases the optimum $\alpha$ reduces sharply and then begins to increase with increasing SNR. For low SNR and high $r$, the outage probability is close to one for any $\alpha$ and optimizing the power allocation does not help. Once SNR increases to the point where $r \simeq C_{p a, n o n-o r t h}(\mathrm{SNR})$, then the outage probability reduces and the optimization result becomes more meaningful. Note that even as $S N R \rightarrow \infty$, both antennas receive non-zero transmit power as the desired operating rate is greater than the maximum achievable rate using a single antenna.

\section{Conclusions}

In this paper, we proposed a new multiphase spreading (MPS) scheme for transmitting independent information on multiple antennas using different phases of Gold codes. The throughput and probability of outage characteristics of MPS in Figures 3 and 4 clearly illustrate its potential. The performance of MPS with larger number of transmit antennas and different constellation sizes using other multiuser detectors should be studied further in future work. In practice, although the antennas at the base station are physically separated, the fading processes might still have some correlation. It has been shown in [18] that MPS also performs well for antennas with correlated fading.

The idea of MPS is to maintain the number of spreading codes per user the same as in the single antenna case, thus it can be easily implemented in $3 \mathrm{G}$ systems. MPS can be easily disabled if the mobile does not support extra processing by reallocating the power between the two phases of spreading codes. It should be noted that the MPS in its 'vanilla' form does not provide any diversity advantage. Only when we superimpose a strong channel code over the modulation do we obtain diversity gains. However, MPS does have inherent ability to separate the different users (as illustrated in Figure $3 \mathrm{~b}$ ) thereby providing some amount of spreading. An interesting research direction is to combine MPS with space-time codes to achieve joint coding, spreading and diversity gains in a multiuser environment.

We also found the optimal power distribution among the transmit antennas with 1 bit of CSI at the transmitter to maximize capacity or minimize outage probability. We found that in the high SNR regime, to maximize throughput, it is best to allocate a substantial portion of the transmit power to the 'worse' antenna: On the other hand to minimize probability of outage most of the power should be allocated to the better antenna. Furthermore, we showed that the throughput maximizing power allocation is robust to feedback errors.

\section{APPENDIX}

\section{Appendix A: EfFECT of CORRELAtion on THROUGHPUT}

Considering the two streams from the two antennas of a particular user, the output of a decorrelator results in two parallel Gaussian channels with correlated noise. We approximate these channels by two BSC's with correlated binary noises. The capacity of two BSC's with crossover probabilities having joint distribution $p_{e 1, e 2}$ equals $C_{\text {joint }}=2+\sum_{x, y \in\{0,1\}} p_{e 1, e 2}(x, y) \log \left(p_{e 1, e 2}(x, y)\right)$. Assuming the two BSC noises to be independent, the capacity is given by $C_{i n d e p}=2+\sum_{x \in\{0,1\}} p_{e 1}(x) \log \left(p_{e 1}(x)\right)+$ $\sum_{x \in\{0,1\}} p_{e 2}(x) \log \left(p_{e 2}(x)\right)$, where $p_{e 1}$ and $p_{e 2}$ are the marginal densities of $p_{e 1, e 2}$. Using numerical evaluations, the difference between $C_{j o i n t}$ and $C_{\text {indep }}$ for $\rho=0,0.25,0.5$ was found to be $0,0.01$ and 0.03 respectively. For the MPS case, using the parameters of Figure 3 , the corresponding correlation is related to the values of $\tilde{R}^{-1}$ and is approximately 0.3 . Hence, the difference in throughput by using the independence assumption is negligible.

\section{REFERENCES}

[1] S. Verdu, Multiuser Detection, Cambridge University Press, 1998.

[2] J. G. Proakis, Digital Communications, Mc-Graw Hill Inc., 1995.

[3] G. J. Foschini and M. J. Gans, "On limits of wireless communications in a fading environment when using multiple antennas," Wireless Personal Communication, 1998.

[4] H. Huang, H. Vishwanathan, and G. J. Foschini, "Achieving high data rates in cdma systems using blast techniques," Proc. GLOBECOM, pp. 2316-2320, 1999.

[5] E. Telatar, "Capacity of multi-antenna Gaussian channels," European Transactions on Telecommunications, pp. 585-595, Nov/Dec 1999.

[6] L. H. Ozarow, S. Shamai, and A. D. Wyner, "Information theoretic considerations for cellular mobile radio," IEEE Tran. Vehicular Technology, vol. 43, no. 2, pp. 359-378, May 1994.

[7] A. Narula, M. J. Lopez, M.D. Trott, and G. Wornell, "Efficient use of side information in multiple-antenna data transmission over fading channels," IEEE JSAC, pp. 1423-1436, Oct. 1998.

[8] V. Tarokh, A. Naguib, N. Seshadri, and A. R. Calderbank, "Combined array processing and space-time coding," IEEE Tran. Info. Thy., pp. 1121-1128, May 1999.

[9] S. M. Alamouti, "A simple transmit diversity technique for wireless communications," IEEE JSAC, vol. 16, no. 8, Oct. 1998.

[10] F. Livingston, V. Chandrasekar, M. Vaya, and J. Cavallaro, "Handset detector architectures for 3G wireless systems," Proc. IEEE Intl. Symp. on Circuits and Systems, 2002.

[11] K. Rohani, "Orthogonal transmit diversity (OTD) for CDMA forward link," Motorola Contribution to ETSI, 1997. 
[12] Samsung, "Time switched transmit diversity (TSTD)," Contribution by TTA to TIA-TTA JSG/01.98.05.13.05, 1998.

[13] Nokia, "Downlink transmit diversity," Contribution to ETSI SMG2, Bocholt, Germany, 51998.

[14] A. Narula, M. D. Trott, and G. Wornell, "Performance limits of coded diversity methods for transmitter antenna arrays," IEEE Tran. Info. Thy., pp. 2418-2433, Nov 1999.

[15] T. M. Cover and J. A. Thomas, Elements of Information Theory, Wiley, New York, 1991

[16] R. G. Gallager, "An inequality on the capacity region of multiaccess multipath channels," in Communications and Cryptography - Two sides of one Tapestry, pp. 129-139. Kluwer, 1994.

[17] A. J. Goldsmith and P. Varaiya, "Capacity of fading channels with channel side information," IEEE Tran. Info. Thy., pp. 1986-1992, Nov. 1997.

[18] D. Rajan, "Spreading and power control for multiple antenna transmit diversity," M.S. thesis, Rice University, Apr. 1999.

\section{ACKNOWLEDGEMENTS}

The authors thank the reviewers for insightful comments and suggestions which improved the quality of the paper.
Oulu, Oulu, Finland, and at the U.S. Air Force Phillips Laboratory, Albuquerque, New Mexico. His research interests include the areas of communication theory, information theory, and their applications with emphasis on multiple-access communications, cellular mobile radio communications, and optical communication networks. works and information theory.

Dinesh Rajan received the B.Tech. degree in Electrical Engineering from Indian Institute of Technology, Madras in 1997. He received his M.S. and Ph.D. degrees in Electrical and Computer Engineering in 1999 and 2002, respectively, from Rice University, Houston, Texas. He joined the Electrical Engineering Department at Southern Methodist University, Dallas, Texas in 2002 , where he is currently an assistant professor. His current research interests include communications theory, wireless net-

Elza Erkip (S'93-M'96) received the Ph.D. and M.S. degrees in Electrical Engineering from Stanford University in 1996 and 1993 respectively, and the B.S. degree in Electrical and Electronic Engineering from Middle East Technical University, Turkey in 1990. Dr. Erkip joined Polytechnic University in Spring 2000, where she is currently an Assistant Professor of Electrical and Computer Engineering. During 1996-1999 she was first a Visiting Assistant Professor and then a Faculty Fellow in the Department of Electrical and Computer Engineering of Rice University. Dr. Erkip received IBM Faculty Partnership Award in 2000 and NSF CAREER award in 2001. Her research interests are in wireless communications, information and communication theory.

Behnaam Aazhang(S'82-M'82-SM'91-F'99) received his B.S. (with highest honors), M.S., and Ph.D. degrees in Electrical and Computer Engineering from University of Illinois at Urbana-Champaign in 1981, 1983, and 1986, respectively. From 1981 to 1985 , he was a Research Assistant in the Coordinated Science Laboratory, University of Illinois. In August 1985, he joined the faculty of Rice University, Houston, Texas, where he is now a Professor in the Department of Electrical and Computer Engineering and the Director of Center for Multimedia Communications. He has been a Visiting Professor at IBM Federal Systems Company, Houston, Texas, the Laboratory for Communication Technology at Swiss Federal Institute of Technology (ETH), Zurich, Switzerland, the Telecommunications Laboratory at University of 